Research Article

\title{
Identification of microRNA signature in different pediatric brain tumors
}

\author{
Marwa Tantawy ${ }^{1}$ D, Mariam G. Elzayat ${ }^{1}$, Dina Yehia $^{1}$ and Hala Taha ${ }^{2,3}$ \\ ${ }^{1}$ Research Department, Children's Cancer Hospital Egypt, Cairo, Egypt. \\ ${ }^{2}$ Pathology Department, Children's Cancer Hospital Egypt, Cairo, Egypt. \\ ${ }^{3}$ Pathology Department, National Cancer Institute, Cairo University, Cairo, Egypt.
}

\begin{abstract}
Understanding pediatric brain tumor biology is essential to help on disease stratification, and to find novel markers for early diagnosis. MicroRNA (miRNA) expression has been linked to clinical outcomes and tumor biology. Here, we aimed to detect the expression of different miRNAs in different pediatric brain tumor subtypes to discover biomarkers for early detection and develop novel therapies. Expression of 82 miRNAs was detected in 120 pediatric brain tumors from fixed-formalin paraffin-embedded tissues, low-grade glioma, high-grade glioma, ependymoma, and medulloblastoma, using quantitative real-time PCR. Low-expression of miR-221, miR-9, and miR-181c/d and over-expression of miR-101, miR-222, miR-139, miR-1827, and miR-34c was found in medulloblastoma; low expression of miR-10a and over-expression of miR-10b and miR-29a in ependymoma; low expression of miR-26a and overexpression of miR-19a/b, miR-24, miR-27a, miR-584, and miR-527 in low-grade glioma. Cox regression showed differential miRNA expression between responders and non-responders. The most specific were miR-10a and miR-29a low expression in LGG non-responders, miR-135a and miR-146b over-expression in ependymoma non-responders, and miR-135b overexpression in medulloblastoma non-responders. MicroRNAs are differentially expressed in subtypes of brain tumors suggesting that they may help diagnosis. A greater understanding of aberrant miRNA in pediatric brain tumors may support development of novel therapies.
\end{abstract}

Keywords: microRNA, pediatric, central nervous system tumors.

Received: January 10, 2017; Accepted: August 21, 2017.

\section{Introduction}

Pediatric brain tumors are the second most common pediatric malignancy, representing about $25 \%$ of all childhood cancers (Boman et al., 2009; Birks et al., 2011). As a result of the high mortality rate and poor prognosis of brain tumors, many studies have focused on the molecular aspects of the disease, including the use of microRNAs (miRNAs) as diagnostic and prognostic markers and even more as therapeutic agents (Wang et al., 2015a; Wang et al., 2015b).

miRNAs are small non-coding RNAs (18-25 nucleotides) that regulate gene expression in many cellular processes by affecting the post-transcriptional regulation (Bartel and Chen, 2004; Jonas and Izaurralde, 2015). During their biogenesis, miRNAs are transcribed to form hairpin structures called pri-miRNAs; the RNase III Drosha enzyme cleaves this structure in the nucleus to form precursor miRNAs (pre-miRNAs). In the cytoplasm, RNase III Dicer enzymes cleave pre-miRNAs to produce mature miRNAs (Lund et al., 2004; Van Wynsberghe et al., 2011). miRNAs are unable to perform their function until the bind-

Send correspondence to Marwa Tantawy. 1 Sekket el imam sayda Zienb, Cairo, Egypt. Email: marwa.tantawy@57357.com. ing with RNA-induced silencing complex occurs (Diederichs and Haber, 2007).

Earlier studies reported that miRNAs have a critical role in key pathways such as cell growth, cell differentiation and apoptosis by controlling their target gene expression. The miRNAs have negative regulation of gene expression by binding the 3' untranslated regions of mRNA of a protein-coding gene. This causes a degradation or blockage of translation of these mRNAs (Zen and Zhang, 2012). A previous study showed the significant role of miR-601 as a putative tumor suppressor gene in pediatric medulloblastoma (MED) (Braoudaki et al., 2014). Other studies have shown that the inhibition of miR-21 in glioblastoma cells increase apoptosis (Faragalla et al., 2012). That makes miRNAs useful biomarkers candidates for diagnosis and prognosis of pediatric brain tumors. In addition, miRNA stability in bodily fluids, functionality in several tissue types, and their capability to detect early phase disease are all useful attributes (D'Urso et al., 2015; Stoicea et al., 2016).

In previous studies, some miRNAs such as miR-129 were strongly down-regulated in brain tumor samples compared to normal tissue, while miR-142-5p and miR-25 were significantly upregulated in all tumor samples compared to 
normal tissue (Birks et al., 2011). More recently, it was demonstrated that miR-19a, miR-15b and miR-106b were significantly up-regulated in MED, while miR-128, miR299-5p, miR-138 were significantly down-regulated compared to normal control samples (Dai et al., 2017). In another study, it was observed that inhibition of miR-106b can induce G1 arrest and apoptosis in MED cells (Li et al., 2015). A differential expression of miR-124 in pediatric pilocytic astrocytoma was found compared to normal brain tissues (Leichter et al., 2017).

The different treatments currently used, such as radiotherapy and chemotherapy, play an essential role in improving outcomes, but finding biomarkers for better diagnosis, prognosis, and management of disease progression is necessary (Tihan et al., 2008; Costa et al., 2011).

To investigate the importance of miRNA expression in pediatric brain tumors, low grade glioma (LGG), ependymoma (EPN), medulloblastoma (MED), and high grade glioma (HGG), their expression levels were characterized in this study using quantitative polymerase chain reaction (qPCR). The discovery of a significant profile of miRNA expression and the ability to distinguish between different histological subtypes will have a great impact on the understanding of pediatric brain tumor biology. Taking into consideration the miRNA connection with clinical outcomes, the growing information arising from laboratory research offers great promise for the advancement of diagnosis, prognosis, and therapy.

\section{Materials and Methods}

\section{Patients and samples}

All tumor specimens were collected retrospectively from patients undergoing surgery at the Children's Cancer Hospital Egypt- 57357 (CCHE) from 2008 to 2015. All studies were conducted in compliance with CCHE-institutional review board regulations (CCHE-IRP \#12-2014). Formalin-fixed paraffin-embedded (FFPE) specimens ( $\mathrm{n}=$ 120) were obtained from the Pathology Department from patients diagnosed with brain tumors (34 LGG, 31 EPN, 30 MED, and $25 \mathrm{HGG}$ ) according to the WHO histological tumor classification (Louis et al., 2007).

\section{RNA extraction}

For FFPE samples, total RNA was isolated from 5-10 $5-\mu \mathrm{m}$ thickness tissue sections, using a miRNeasy FFPE kit (Qiagen, Hilden, Germany) according to the manufacturer's instructions. Total RNA quantity and quality were evaluated using a spectrophotometer (Nanodrop ND-1000, Thermo Scientific, Wilmington, USA).

\section{Reverse transcription and quantitative polymerase chain reaction ( $q R C R)$}

Total RNA was reverse transcribed using a miScript RT kit (Qiagen). Reactions were incubated at $37^{\circ} \mathrm{C}$ for $1 \mathrm{~h}$ followed by inactivation of the reaction by incubation at $95{ }^{\circ} \mathrm{C}$ for $10 \mathrm{~min}$. For miRNA expression profiling, the primers used for qPCR were obtained from Invitrogen. One microliter of diluted RT product was used (equivalent to $10 \mathrm{ng}$ ) as a template in a $10-\mu \mathrm{L}$ PCR reaction containing $1 \mathrm{X}$ SYBR Green master mix (Qiagen), 200 nM miRNAspecific forward primer, and $200 \mathrm{nM}$ universal primer. The conditions for qPCR were as follows: $95{ }^{\circ} \mathrm{C}$ for $10 \mathrm{~min}$, followed by 40 cycles of $95{ }^{\circ} \mathrm{C}$ for $15 \mathrm{~s}, 55^{\circ} \mathrm{C}$ for $30 \mathrm{~s}$, and $72{ }^{\circ} \mathrm{C}$ for $30 \mathrm{~s}$. All qPCR reactions were performed on a QuantStudio 6 flex real-time PCR system (Applied Biosystems, Foster City, CA, USA).

The normalized relative expression levels of miRNAs were calculated using the delta cycle threshold (dCT) method, all CT values above or equal to 35 were replaced with 35 before calculating the mean of the remaining CT values. The mean $\mathrm{CT}$ value was calculated for each sample $(\Delta \mathrm{CT}=$ CT sample - CT mean expression of individual miRNA plate) (Mestdagh et al., 2009).

\section{Statistical analysis}

Statistical evaluation was done using GraphPad Prism software version 5.01 (GraphPad, Inc., San Diego, CA, USA) and the SPSS win statistical package version 18. Numerical data are reported as mean \pm standard deviation (SD), median, and range. Qualitative data are reported as frequency and percentage. The normal distribution of variables was assessed prior to selecting the tests for statistical analyses. The values of miRNAs were analyzed using either nonparametric Kruskal-Wallis or unpaired Student $t$ tests. The relationship between patient outcomes and miRNA expression profiles was analyzed using MannWhitney $U$ tests. Stepwise backward multivariable logistic regression was performed. The survival rates were analyzed using log-rank analysis. A $P$ value of less than 0.05 was considered significant.

\section{Results}

\section{Patient samples}

miRNA expression was measured using quantitative RT-PCR in 120 samples from pediatric brain tumors (34 LGG, $31 \mathrm{EPN}, 30 \mathrm{MED}$, and $25 \mathrm{HGG}$ ); patients with the same disease were treated with the same protocol. The median age of LGG patients was 8.1 years, for EPN patients 3 years, for MED patients 6 years, and for HGG patients 9.7 years. The male/female ratio for LGG patients was 1:0, for EPN patients 1:1.7, for MED patients 3:1, and for HGG patients 1:3. The clinical and pathological characterization of patients enrolled in the present study is shown in Table 1.

Three-year overall survival for LGG, EPN, MED, and HGG respectively was $93.8 \%$ (95\% CI 63.21-71.84), 67.7\% (95\% CI 50.9-72.9), 75.3\% (95\% CI 45.962.3), and $24.4 \%$ (95\% CI 26.254). Three-year event-free survival for LGG, EPN, MED, and HGG respectively was $90.9 \%$ (95\% 
Table 1 - Clinicopathological features of pediatric brain tumor patients enrolled in this study.

\begin{tabular}{|c|c|c|c|c|c|}
\hline & & LGG & EPN & MED & HGG \\
\hline & & $\mathrm{n}=34$ & $\mathrm{n}=31$ & $\mathrm{n}=30$ & $\mathrm{n}=25$ \\
\hline \multirow[t]{3}{*}{ Age } & Mean & 8.6 & 4.9 & 6.7 & 8.7 \\
\hline & Median & 8.1 & 3 & 6 & 9.7 \\
\hline & Range & $2.8-16$ & $0.7-16.6$ & $2.8-14$ & $2.1-16.6$ \\
\hline \multirow[t]{2}{*}{ Gender } & Female & $19(55.9 \%)$ & $8(25.8 \%)$ & $16(53.3 \%)$ & $14(56 \%)$ \\
\hline & Male & $15(44.1 \%)$ & $23(74.2 \%)$ & $14(46.7 \%)$ & $11(44 \%)$ \\
\hline \multirow[t]{3}{*}{ Age category } & $\leq 1$ year & 0 & $2(6.5 \%)$ & 0 & 0 \\
\hline & $>1$ year and $<10$ years & $23(67.6 \%)$ & $24(77.4 \%)$ & $25(83.3 \%)$ & $16(64 \%)$ \\
\hline & $\geq 10$ years & $11(32.4 \%)$ & $5(16.1 \%)$ & $5(16.7 \%)$ & $9(36 \%)$ \\
\hline \multirow[t]{3}{*}{ Tumor size } & $\leq 5 \mathrm{~cm}$ & $21(61.8 \%)$ & $15(48.4 \%)$ & $21(70 \%)$ & $8(32 \%)$ \\
\hline & $>5 \mathrm{~cm}$ & $12(35.3 \%)$ & $15(48.4 \%)$ & $8(26.7 \%)$ & $14(56 \%)$ \\
\hline & Unknown & $1(2.9 \%)$ & $1(3.2 \%)$ & $1(3.3 \%)$ & $3(12 \%)$ \\
\hline \multirow[t]{4}{*}{ Grade (WHO)* } & I & $34(100 \%)$ & 0 & 0 & 0 \\
\hline & II & 0 & 0 & 0 & 0 \\
\hline & III & 0 & $31(100 \%)$ & 0 & $6(24 \%)$ \\
\hline & IV & 0 & 0 & $30(100 \%)$ & $19(76 \%)$ \\
\hline \multirow[t]{2}{*}{ Metastasis at presentation } & No & $34(100 \%)$ & $31(100 \%)$ & $18(60 \%)$ & $21(84 \%)$ \\
\hline & Yes & 0 & 0 & $12(40 \%)$ & $4(16 \%)$ \\
\hline \multirow[t]{2}{*}{ Risk } & High & 0 & $31(100 \%)$ & $30(100 \%)$ & $25(100 \%)$ \\
\hline & Low & $34(100 \%)$ & 0 & 0 & 0 \\
\hline \multirow[t]{4}{*}{ Patient response } & Complete Remission & $26(76.5 \%)$ & $13(41.9 \%)$ & $24(80 \%)$ & $3(12 \%)$ \\
\hline & Partial Remission & $5(14.7 \%)$ & $2(6.5 \%)$ & 0 & $2(8 \%)$ \\
\hline & No Response & 0 & 0 & $1(3.3 \%)$ & $2(8 \%)$ \\
\hline & Progressive Disease & $3(8.8 \%)$ & $16(51.6 \%)$ & $5(16.7)$ & $18(72 \%)$ \\
\hline \multirow[t]{2}{*}{ Event } & No (median 38 Months) & $30(88.2 \%)$ & $15(48.4 \%)$ & $22(73.3 \%)$ & $5(20 \%)$ \\
\hline & Yes & $4(11.8 \%)$ & $16(51.6 \%)$ & $8(26.7 \%)$ & $20(80 \%)$ \\
\hline \multirow[t]{2}{*}{ Survival status } & Dead & $2(5.9 \%)$ & $10(32.3 \%)$ & $7(23.3 \%)$ & $16(64 \%)$ \\
\hline & Alive & $32(94.1 \%)$ & $21(67.7 \%)$ & $23(76.7 \%)$ & $9(36 \%)$ \\
\hline
\end{tabular}

LGG, Low grade glioma; EPN, Ependymoma; MED, Medulloblastoma; HGG, High grade glioma; and n, number *Based on standard WHO (World Health Organization) classification for brain tumors.

CI 57.270$), 43.3 \%(95 \%$ CI 3358.5$), 72.6 \%(95 \%$ CI 4461$)$, and $15.6 \%(95 \%$ CI 14.8137.2).

\section{Selection of miRNA for tumor tissue profiling}

For our study, we selected the most significant miRNAs expressed in brain tumors compared with normal cells from previous studies. Other miRNAs were selected to distinguish between different subtypes of brain tumors and few were selected specifically for prognosis. Therefore, we identified a panel of 82 miRNAs, which are provided in Table $\mathrm{S} 1$.

\section{Profiling of 82 miRNA expression patterns in pediatric brain tumors}

Most miRNAs were expressed in all subtypes with no significant change. The most significantly over-expressed miRNAs were miR-19a/b, miR-24, miR-27a, miR-584, and
miR-527 in LGG, miR-10b, and miR-29a in EPN, and miR-101, miR-222, miR-139, miR-1827, and miR-34c in MED. The most significantly under-expressed miRNAs were miR-26a in LGG, miR-10a in EPN, and miR-221, miR-9, and miR-181c/d in MED. Lower dCT scores were observed in subtypes representing the higher level of expression of selected miRNAs; P values of each subtype compared with other types are shown in Tables S2-S4.

\section{miRNAs significantly associated with patient response to treatment}

To elucidate whether the pre-treatment miRNA expression profile is related with the patient's response to chemotherapy treatment, we compared the normalized pre-treatment expression profile of the 82 miRNAs in a group of chemotherapy responders with that of non-responders in LGG and EPN groups. Patients with LGG and 
EPN are classified as responders and non-responders according to the following; definitions: patients with complete response (CR) were considered as responders, while patients with partial remission (PR) and progressive disease (PD) were categorized as non-responders (from Children's Cancer Group CCG-A9952, CCG-9942 respectively, according to the roadmap treatment for LGG, EPN, MED and HGG (Figures S1-S4). Out of the 34 LGG patients investigated in this study, 26 were classified as responders while eight were non-responders. Out of the 31 EPN patients, 13 were classified as responders while 18 were non-responders (Figures S5-S7).

The relationship between miRNAs and treatment response was addressed by controlling for age, gender and grade using a multivariate Cox regression analysis as shown in Table 2. Low expression of miR-10a and miR-29a and high expression of miR-361-5p, miR- 617, miR-92a, miR-527, and miR-206 were detected in LGG non-responders and identified as independent factors for treatment response. We also identified miR-135a and miR-146b over-expression in EPN non-responders. The data suggest that these miRNAs could be used as biomarkers and predictors for treatment response likelihood (Figures 1 and 2).

\section{Discussion}

miRNAs have been identified as critical regulators of tumorigenesis in a variety of cancers, but their role in pedi- atric brain cancers has only recently been recognized. To explore the importance of miRNAs in pediatric brain tumors, FFPE specimens of LGG, EPN, MED, and HGG were selected for miRNA expression using RT-qPCR. We identified several miRNAs expressed differentially between different pathological subtypes.

In our study, miR-10b was overexpressed in EPN compared to other subtypes. A previous study, however, showed significant alteration in the expression of miR-10b in HGG compared to LGG cell lines (Sasayama et al., 2009; Visani et al., 2014a). Other studies suggested that the overexpression in miR-10b in cancer cells may be correlated with an increase in hypoxia (Haque et al., 2011). A study investigating predictive markers to help in glioma prognosis found that the increase in miR-10b expression in adult patients with glioma, including high and low grade gliomas, is associated with poor prognosis (Zhang et al., 2016).

miR-26a was found decreased in LGG compared to other high-grade subtypes; this is in accordance with a recent study that showed that miR-26a-5p levels decreased with glioma grade (Sharma et al., 2016). We observed a low expression of miR-9 in MED, in accordance with recently reported studies in MED and neuroblastoma (Laneve et al., 2007; Ferretti et al., 2009). In contrast with another study that reported down-regulation of miR-101 in FFPE of HGG but not in LGG (Visani et al., 2014b), we found an overexpression of miR-101 in MED patients.

Table 2 - Clinicopathological features of pediatric brain tumor patients according to treatment response.

\begin{tabular}{|c|c|c|c|c|c|c|c|c|c|}
\hline & & \multicolumn{2}{|c|}{ LGG } & \multicolumn{2}{|c|}{ EPN } & \multicolumn{2}{|c|}{ MED } & \multicolumn{2}{|c|}{ HGG } \\
\hline & & $\mathrm{R}$ & NR & $\mathrm{R}$ & NR & $\mathrm{R}$ & NR & $\mathrm{R}$ & NR \\
\hline No. & & 26 & 8 & 13 & 18 & 24 & 6 & 3 & 22 \\
\hline \multirow[t]{2}{*}{ Gender } & Male & 11 & 4 & 6 & 17 & 12 & 2 & 1 & 10 \\
\hline & Female & 15 & 4 & 7 & 1 & 12 & 4 & 2 & 12 \\
\hline \multirow[t]{3}{*}{ Age } & $\leq 1$ year & 0 & 0 & 1 & 1 & 0 & 0 & 0 & 0 \\
\hline & $>1$ year and $<10$ years & 18 & 5 & 9 & 15 & 21 & 4 & 3 & 13 \\
\hline & $\geq 10$ years & 8 & 3 & 3 & 2 & 3 & 2 & 0 & 9 \\
\hline \multirow[t]{4}{*}{ Grade (WHO) } & I & 26 & 8 & 0 & 0 & 0 & 0 & 0 & 0 \\
\hline & II & 0 & 0 & 0 & 0 & 0 & 0 & 0 & 0 \\
\hline & III & 0 & 0 & 13 & 18 & 0 & 0 & 3 & 3 \\
\hline & IV & 0 & 0 & 0 & 0 & 24 & 6 & 0 & 19 \\
\hline \multirow[t]{3}{*}{ Tumor size } & $\leq 5 \mathrm{~cm}$ & 16 & 5 & 7 & 8 & 17 & 4 & 1 & 7 \\
\hline & $>5 \mathrm{~cm}$ & 9 & 3 & 5 & 10 & 6 & 2 & 1 & 13 \\
\hline & Unknown & 1 & 0 & 1 & 0 & 1 & 0 & 1 & 2 \\
\hline \multirow[t]{2}{*}{ Survival status } & Dead & 1 & 1 & 0 & 10 & 2 & 5 & 0 & 16 \\
\hline & Alive & 25 & 7 & 13 & 8 & 22 & 1 & 3 & 6 \\
\hline \multirow[t]{2}{*}{ Event } & Yes & 2 & 2 & 0 & 16 & 2 & 6 & 0 & 20 \\
\hline & No & 24 & 6 & 13 & 2 & 22 & 0 & 3 & 2 \\
\hline
\end{tabular}

LGG, Low grade glioma; EPN, Ependymoma; MED, Medulloblastoma; HGG, High grade glioma; and n, number R, Responder; NR, Non-Responder. *Based on standard WHO (World Health Organization) classification for brain tumours. 

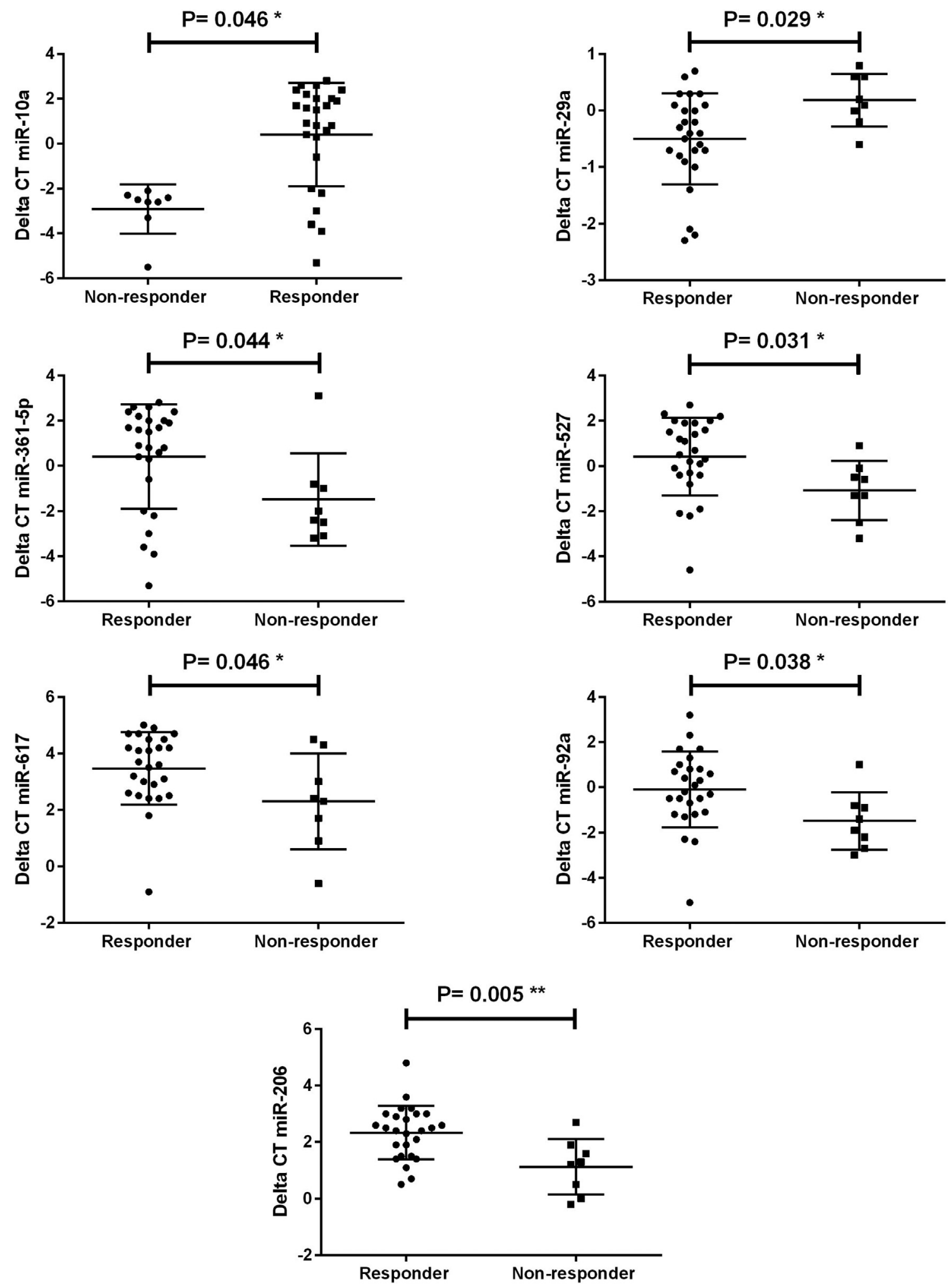

Figure 1 - miRNAs significantly deregulated in response to treatment in LGG.

In our study, miR-10b was overexpressed not only in glioma tissues but also in glioma cell lines

This study showed that there are specific miRNAs in each subtype differentially expressed in responders and non-responders to chemotherapy: in LGG there was a lower expression of miR-10a in non-responders compared to responders, while previous studies have shown miR-10a regulating $\mathrm{T}$ follicular helper maturation (Paladini et al., 

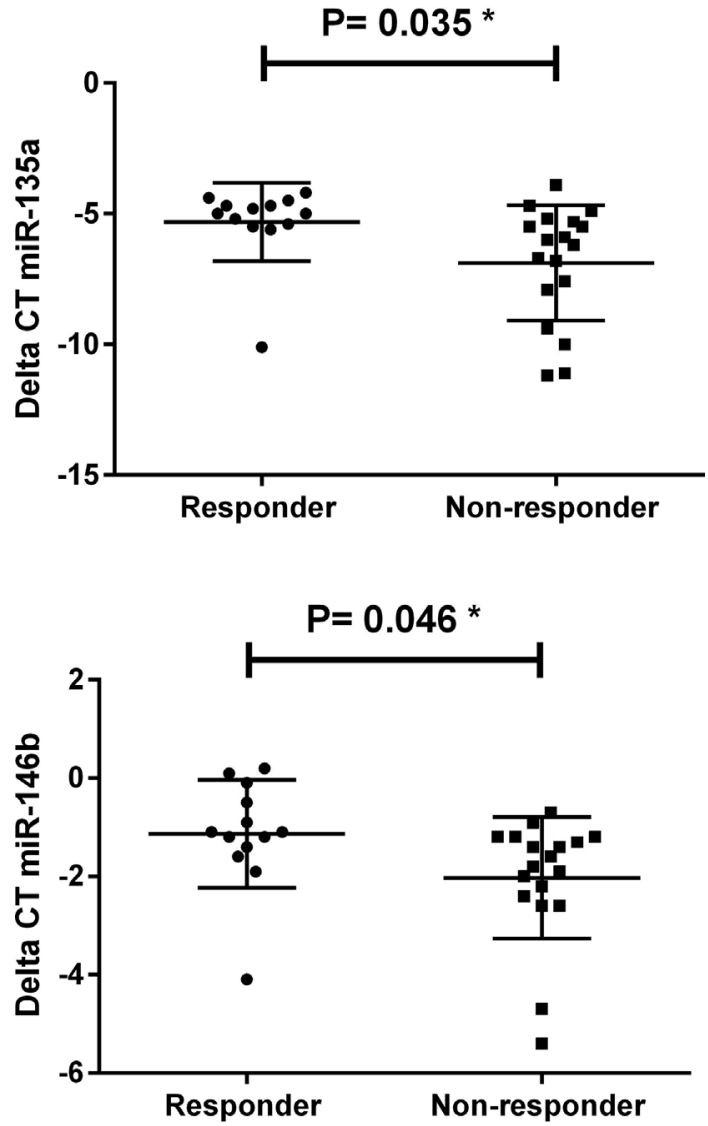

Figure 2 - miRNAs significantly deregulated in response to treatment in EPN.

2016). Significantly lower expression was also detected for miR-29a in LGG non-responders compared to responders, while a previous study identified miR-29 as a negative regulator of the B7-H3 protein, which acts as a surface immunomodulatory glycoprotein inhibiting natural killer (NK) and T-cell functions (Xu et al., 2009). A previous study showed an inverse correlation between miR-29 and B7-H3 in solid tumors in cell line experiments (Wang et al., 2013); another study showed the role of miR-29 in the promotion of anti-tumor immunity mediated by NK and T-cells (Filipazzi et al., 2012).

In EPN patients, overexpression of miR-146a was found in non-responders compared to responders, while another study showed the role of miR-146a in inhibiting $\mathrm{T}$ cytotoxic immune responses (Liang et al., 2015).

Significant overexpression of miR-135a and miR$135 \mathrm{~b}$ was detected in EPN and MED in non-responders, respectively. A previous study showed that overexpression of $\mathrm{miR}-135 \mathrm{a} / \mathrm{b}$ increased the resistance of lung cell lines treated with cisplatin (Zhou et al., 2013).

Certain miRNAs have been correlated with outcomes of brain tumors, however these were not significant in this study. Another study found that the decrease of miR-124 may be correlated with malignant progression and poor prognosis in adult patients with gliomas (Chen et al., 2015).
A positive correlation was found between the overexpression of miR-219 and overall survival in pediatric patients with MED (Pezuk et al., 2017). In addition, the over expression of miR-19a and miR-106b showed a significant correlation with tumor grade III of EPN (Zakrzewska et al., 2016).

Additional studies with larger cohorts are needed to confirm the potential biomarkers reported in this pilot study. Pediatric brain tumors have unique miRNA profiles and the characterization of miRNA expression in serum may be an interesting follow-up study. A greater understanding of the aberrant expression of miRNAs in brain tumors of different subtypes may aid in the discovery of novel therapeutic methods.

\section{Acknowledgments}

The authors thank the Science and Technology Development Fund for financial support (project ID \# 6014), and the Central Nervous System Clinical Research Team in Children's Cancer Hospital Egypt-57357 for their support with clinical data. The authors also thank the Radiology Department at Children's Cancer Hospital Egypt for their assistance.

\section{References}

Bartel DP and Chen C-Z (2004) Micromanagers of gene expression: The potentially widespread influence of metazoan microRNAs. Nat Rev Genet 5:396-400.

Birks DK, Barton VN, Donson AM, Handler MH, Vibhakar R and Foreman NK (2011) Survey of microRNA expression in pediatric brain tumors. Pediatr Blood Cancer 56:211-216.

Boman KK, Hovén E, Anclair M, Lannering B and Gustafsson G (2009) Health and persistent functional late effects in adult survivors of childhood CNS tumours: A population-based cohort study. Eur J Cancer 45:2552-2561.

Braoudaki M, Lambrou GI, Giannikou K, Milionis V, Stefanaki K, Birks DK, Prodromou N, Kolialexi A, Kattamis A, Spiliopoulou CA, et al. (2014) MicroRNA expression signatures predict patient progression and disease outcome in pediatric embryonal central nervous system neoplasms. J Hematol Oncol 7:96.

Chen T, Wang X, Li C and Xu S (2015) Downregulation of microRNA-124 predicts poor prognosis in glioma patients. Neurol Sci 36:131-135.

Costa FF, Bischof JM, Vanin EF, Lulla RR, Wang M, Sredni ST, Rajaram V, de Fátima Bonaldo M, Wang D, Goldman S, et al. (2011) Identification of microRNAs as potential prognostic markers in ependymoma. PLoS One 6:e25114.

Dai J, Li Q, Bing Z, Zhang Y, Niu L, Yin H, Yuan G and Pan Y (2017) Comprehensive analysis of a microRNA expression profile in pediatric medulloblastoma. Mol Med Rep 15:4109-4115.

Diederichs S and Haber DA (2007) Dual role for Argonautes in microRNA processing and posttranscriptional regulation of microRNA expression. Cell 131:1097-1108.

D'Urso IP, D’Urso FO, Damiano GC, Mezzolla V, Storelli C and Marsigliante S (2015) miR-15b and miR-21 as circulating 
biomarkers for diagnosis of glioma. Curr Genomics 16:304-311.

Faragalla H, Youssef YM, Scorilas A, Khalil B, White NMA, Mejia-Guerrero S, Khella H, Jewett MAS, Evans A, Lichner Z, et al. (2012) The clinical utility of miR-21 as a diagnostic and prognostic marker for renal cell carcinoma. JMDI 14:385-392.

Ferretti E, De Smaele E, Po A, Di Marcotullio L, Tosi E, Espinola MSB, Di Rocco C, Riccardi R, Giangaspero F, Farcomeni A, et al. (2009) MicroRNA profiling in human medulloblastoma. Int J Cancer 124:568-577.

Filipazzi P, Huber V and Rivoltini L (2012) Phenotype, function and clinical implications of myeloid-derived suppressor cells in cancer patients. Cancer Immunol Immunother 61:255-263.

Haque I, Banerjee S, Mehta S, De A, Majumder M, Mayo MS, Kambhampati S, Campbell DR and Banerjee SK (2011) Cysteine-rich 61-Connective Tissue Growth Factor-nephroblastoma-overexpressed 5 (CCN5)/Wnt-1-induced Signaling Protein-2 (WISP-2) regulates microRNA-10b via Hypoxia-inducible Factor-1 -TWIST signaling networks in human breast cancer cells. J Biol Chem 286:43475-43485.

Jonas S and Izaurralde E (2015) Towards a molecular understanding of microRNA-mediated gene silencing. Nat Rev Genet 16:421-433.

Laneve P, Di Marcotullio L, Gioia U, Fiori ME, Ferretti E, Gulino A, Bozzoni I and Caffarelli E (2007) The interplay between microRNAs and the neurotrophin receptor tropomyosinrelated kinase $\mathrm{C}$ controls proliferation of human neuroblastoma cells. Proc Natl Acad Sci U S A 104:7957-7962.

Leichter AL, Sullivan MJ, Eccles MR and Chatterjee A (2017) MicroRNA expression patterns and signalling pathways in the development and progression of childhood solid tumours. Mol Cancer 16:15.

Li KK-W, Xia T, Ma FMT, Zhang R, Mao Y, Wang Y, Zhou L, Lau K-M and Ng H-K (2015) miR-106b is overexpressed in medulloblastomas and interacts directly with PTEN. Neuropathol Appl Neurobiol 41:145-164.

Liang Y, Pan H-F and Ye D-Q (2015) MicroRNAs function in CD8+T cell biology. J Leukoc Biol 97:487-497.

Louis DN, Ohgaki H, Wiestler OD, Cavenee WK, Burger PC, Jouvet A, Scheithauer BW and Kleihues P (2007) The 2007 WHO classification of tumours of the central nervous system. Acta Neuropathol 114:97-109.

Lund E, Güttinger S, Calado A, Dahlberg JE and Kutay U (2004) Nuclear export of microRNA precursors. Science 303:95-98.

Mestdagh P, Van Vlierberghe P, De Weer A, Muth D, Westermann F, Speleman F and Vandesompele J (2009) A novel and universal method for microRNA RT-qPCR data normalization. Genome Biol 10:R64.

Paladini L, Fabris L, Bottai G, Raschioni C, Calin GA and Santarpia L (2016) Targeting microRNAs as key modulators of tumor immune response. J Exp Clin Cancer Res 35:103.

Pezuk JA, Brassesco MS, de Oliveira RS, Machado HR, Neder L, Scrideli CA and Tone LG (2017) PLK1-associated microRNAs are correlated with pediatric medulloblastoma prognosis. Child's Nerv Syst 33:609-615.

Sasayama T, Nishihara M, Kondoh T, Hosoda K and Kohmura E (2009) MicroRNA-10b is overexpressed in malignant glio- ma and associated with tumor invasive factors, uPAR and RhoC. Int J Cancer 125:1407-1413.

Sharma V, Purkait S, Takkar S, Malgulwar PB, Kumar A, Pathak $\mathrm{P}$, Suri V, Sharma MC, Suri A, Kale SS, et al. (2016) Analysis of EZH2: micro-RNA network in low and high grade astrocytic tumors. Brain Tumor Pathol 33:117-128.

Stoicea N, Du A, Lakis DC, Tipton C, Arias-Morales CE and Bergese SD (2016) The miRNAjourney from theory to practice as a CNSbiomarker. Front Genet 7:11.

Tihan T, Zhou T, Holmes E, Burger PC, Ozuysal S and Rushing EJ (2008) The prognostic value of histological grading of posterior fossa ependymomas in children: A Children's Oncology Group study and a review of prognostic factors. Mod Pathol 21:165-177.

Van Wynsberghe PM, Chan S-P, Slack FJ and Pasquinelli AE (2011) Analysis of microRNA expression and function. Methods Cell Biol 106:219-252.

Visani M, de Biase D, Marucci G, Cerasoli S, Nigrisoli E, Bacchi Reggiani ML, Albani F, Baruzzi A, Pession A, Brandes AA, et al. (2014a) Expression of 19 microRNAs in glioblastoma and comparison with other brain neoplasia of grades I-III. Mol Oncol 8:417-430.

Visani M, de Biase D, Marucci G, Cerasoli S, Nigrisoli E, Bacchi Reggiani ML, Albani F, Baruzzi A, Pession A and PERNO study group (2014b) Expression of 19 microRNAs in glioblastoma and comparison with other brain neoplasia of grades I-III. Mol Oncol 8:417-430.

Wang F, Ren X and Zhang X (2015a) Role of microRNA-150 in solid tumors. Oncol Lett 10:11-16.

Wang M, Deng X, Ying Q, Jin T, Li M and Liang C (2015b) MicroRNA-224 targets ERG2 and contributes to malignant progressions of meningioma. Biochem Biophys Res Commun 460:354-361.

Wang Y, Zhang X, Li H, Yu J and Ren X (2013) The role of miRNA-29 family in cancer. Eur J Cell Biol 92:123-128.

Xu H, Cheung IY, Guo H-F and Cheung N-KV (2009) MicroRNA miR-29 modulates expression of immunoinhibitory molecule B7-H3: potential implications for immune based therapy of human solid tumors. Cancer Res 69:6275-6281.

Zakrzewska M, Fendler W, Zakrzewski K, Sikorska B, Grajkowska W, Dembowska-Baginska B, Filipek I, Stefanczyk L and Liberski PP (2016) Altered microRNA expression is associated with tumor grade, molecular background and outcome in childhood infratentorial ependymoma. PLoS One 11:e0158464.

Zen K and Zhang C (2012) Circulating microRNAs: A novel class of biomarkers to diagnose and monitor human cancers. Med Res Rev 326-348.

Zhang X, Cheng J, Fu L and Li Q (2016) Overexpression of tissue microRNA10b may help predict glioma prognosis. J Clin Neurosci 29:59-63.

Zhou L, Qiu T, Xu J, Wang T, Wang J, Zhou X, Huang Z, Zhu W, Shu Y and Liu P (2013) miR-135a/b Modulate cisplatin resistance of human lung cancer cell line by targeting MCL1. Pathol Oncol Res 19:677-683.

\section{Supplementary material}

The following online material is available for this article: Table S1 - List of miRNA sequences used in this study.

Table S2 - dCT values of differently expressed miRNAs in MED compared to other subtypes (EPN, LGG, and HGG). 
Table S3 - dCT values of differently expressed miRNAs in EPN compared to other subtypes (MED, LGG, and HGG). Table S4 - dCT values of differently expressed miRNAs in LGG compared to other subtypes (MED, EPN, and HGG). Figure S1 - Roadmap treatment of LGG. Figure S2 - Roadmap treatment of EPN. Figure S3 - Roadmap treatment of MED. Figure S4 - Roadmap treatment of HGG.

Figure S5 - MRI images for a representative LGG case with a good therapy response.

\section{Erratum}

In the article "Identification of microRNA signature in different pediatric brain tumors" with DOI number 10.1590/1678-4685-GMB-2016-0334, published in the journal Genetics and Molecular Biology, 41(1):27-34, on page 28 the confidence intervals stated in section Patient samples were reported wrongly.

\section{Instead of:}

Three-year overall survival for LGG, EPN, MED, and HGG respectively was $93.8 \%$ (95\% CI 63.21-71.84), $67.7 \%$ (95\% CI 50.9-72.9), 75.3\% (95\% CI 45.962.3), and $24.4 \%$ (95\% CI 26.254). Three-year event-free survival for LGG, EPN, MED, and HGG respectively was $90.9 \%$ (95\%
Figure S6 - MRI images for a representative LGG, for a non-responder case.

Figure S7 - MRI images for a representative EPN case with a good therapy response case.

Associate Editor: Anamaria Aranha Camargo

License information: This is an open-access article distributed under the terms of the Creative Commons Attribution License (type CC-BY), which permits unrestricted use, distribution and reproduction in any medium, provided the original article is properly cited.

CI 57.270), 43.3\% (95\% CI 3358.5), 72.6\% (95\% CI 4461), and $15.6 \%$ (95\% CI 14.8137.2).

Correct is:

Three-year overall survival for LGG, EPN, MED, and $\mathrm{HGG}$ respectively was $93.8 \%$ (95\% CI 85.57-102.03), $67.7 \% \quad(95 \% \quad$ CI $\quad 51.24-84.16), \quad 75.3 \% \quad(95 \% \quad$ CI 59.04-91.56), and 40.7\% (95\% CI 20.52-60.88). Three-year event-free survival for LGG, EPN, MED, and HGG respectively was $90.9 \%$ (95\% CI 81.1-100.7), 49.5\% (95\% CI 30.88-68.12), 72.6\% (95\% CI 56.34-88.86), and $28 \%$ (95\% CI 10.36-45.64).

The authors informed that the correction does not affect the general results and conclusions of the study. 